\title{
Etiology and Related Factors of Postoperative Delirium in Orthopedic Surgery
}

\author{
Kyung-Jin Song, MD, Jong-Hyun Ko, MD, Tae-Young Kwon, MD, Byung-Wan Choi, MD* \\ Department of Orthopedic Surgery, Institute for Medical Science, Chonbuk National University Medical School, Jeonju, \\ ${ }^{*}$ Department of Orthopedic Surgery, Haeundae Paik Hospital, Inje University Medical School, Busan, Korea
}

\begin{abstract}
Background: Delirium is a serious complication for elderly patients after orthopedic surgery. The purpose of this study was to assess the etiology and related factors of delirium after orthopedic surgery in Korea.

Methods: We retrospectively reviewed the medical records of 3,611 patients over 50 years who had orthopedic surgery. The age of patients (50s, 60s, 70s, and > 80s), type of anesthesia (general, spinal, and local), operation time (more than 2 hours vs. less than 2 hours), surgical site (spine, hip, knee, or others), and etiology (trauma or disease) were compared to determine possible risk factors of delirium after orthopedic surgery.

Results: Of 3,611 patients, 172 (4.76\%) were diagnosed with delirium after orthopedic surgery. Postoperative delirium occurred in $1.18 \%$ in their 50 s, $3.86 \%$ in their 60 s, $8.49 \%$ in their 70 s, and $13.04 \%$ in $>80$ s ( $p<0.001)$. According to anesthesia type, $6.50 \%$ of postoperative delirium occurred after general anesthesia, $0.77 \%$ after spinal anesthesia, and $0.47 \%$ after local anesthesia $(p<$ 0.001). More than 2 hours of operation was associated with higher occurrence of delirium than less than 2 hours was $(5.88 \%$ vs. $4.13 \%, p=0.017)$. For the etiology, $8.17 \%$ were trauma cases and $3.02 \%$ were disease $(p<0.001)$. Postoperative delirium occurred in 22 of 493 patients (4.46\%) after spine surgery, 18 of 355 patients (5.07\%) after hip surgery, 17 of 394 patients $(4.31 \%)$ after knee surgery, and 15 of 1,145 patients $(1.31 \%)$ after surgery at other sites $(p<0.001)$.

Conclusions: Postoperative delirium was more common in older patients who had surgery under general anesthesia, whose surgery took more than 2 hours, and who were hospitalized through the emergency room.

Keywords: Delirium, Orthopedic surgery, Etiology, Risk factor
\end{abstract}

Delirium is characterized by an acute and fluctuating impairment of consciousness that is accompanied by disturbances in attention, cognition, and perception. ${ }^{1-4)}$ Early diagnosis and treatment of delirium can reduce length of stay, in-hospital morbidity, and health care costs. Previous studies have identified a number of risk factors of postoperative delirium, including preexisting dementia, old age, functional impairment, greater comorbidities, and psycho-

Received February 15, 2019; Accepted March 20, 2019

Correspondence to: Byung-Wan Choi, MD

Department of Orthopedic Surgery, Haeundae Paik Hospital, Inje University Medical School, 875 Haeun-daero, Haeundae-gu, Busan 48108, Korea

Tel: +82-51-797-0240, Fax: +82-51-797-0249

E-mail: alla1013@naver.com pathological symptoms. $^{2)}$

Postoperative delirium is a serious complication for elderly patients because it can cause functional decline, longer hospitalization, and institutionalization. ${ }^{2,5-7)}$ Furthermore, patients with delirium are at risk for dangerous complications, such as falls, aspiration pneumonia, and accidental removal of lines or tubes in the hospital. Considering these serious complications, it is essential to recognize the risk factors of delirium to prevent it after surgery. The additional risk factors of delirium after orthopedic surgery include the use of general anesthesia, surgical duration $>3$ hours, intraoperative hypercapnia and hypotension, preoperative affective dysfunction, and postoperative sleep disorders. ${ }^{8)}$

Most of the published articles about the postoperative delirium are based on an evaluation of Western data. 
By contrast, there is lack of information on the etiology of delirium and clinical results in patients with postoperative delirium in Korea. The purpose of this study was to assess the etiology of delirium after orthopedic surgery and to determine related factors of postoperative delirium in a Korean population.

\section{METHODS}

We retrospectively reviewed the medical records of 3,611 patients aged 50 years or older who had orthopedic surgery at our institution from January 1, 2014, to July 31, 2016. This study was conducted according to the Helsinki Declaration and approved by Institutional Review Board of Chonbuk National University Hospitl (IRB No. 201704-024). Written informed consent to participate in the study was obtained from all patients. There were 1,785 men and 1,826 women with a mean age of $65.7 \pm 9.5$ years (range, 50 to 108 years). The age of patients, type of anesthesia (general, spinal, or local), operation time, surgical site, and etiology (trauma or disease) were examined to determine the possible risk factors of delirium after orthopedic surgery.

Patients were divided into four age groups: 50 to 59 years (1,273 patients), 60 to 69 years (1,167 patients), 70 to 79 years ( 895 patients), and 80 years or older (276 patients). Patients were also categorized according to the type of anesthesia: 2,538 patients had orthopedic surgery

\section{Table 1. Incidence of Postoperative Delirium According to Age}

\begin{tabular}{|ccc|}
\hline Age $(y r)$ & No. of patients & Postoperative delirium \\
\hline $50-59$ & 1,273 & $15(1.18)$ \\
$60-69$ & 1,167 & $45(3.86)$ \\
$70-79$ & 895 & $76(8.49)$ \\
$>80$ & 276 & $36(13.04)$ \\
\hline
\end{tabular}

Values are presented as number (\%).

\begin{tabular}{lcc|}
$\begin{array}{c}\text { Table 2. Incidence of Postoperative } \\
\text { Anesthesia Type }\end{array}$ & Delirium According to \\
\hline Anesthesia & No. of patients & Postoperative delirium \\
\hline General & 2,538 & $165(6.50)$ \\
\hline Spinal & 648 & $5(0.77)$ \\
\hline Local & 425 & $2(0.47)$ \\
\hline
\end{tabular}

Values are presented as number (\%). under general anesthesia, 648 had spinal anesthesia, and 425 had local anesthesia. In addition, 1,224 patients were hospitalized through the emergency room, and 2,387 patients were hospitalized through the outpatient clinic. Of the 3,611 patients, 1,310 patients had orthopedic surgery that lasted more than 2 hours. To assess the morbidity rate due to postoperative delirium according to the surgical site, we divided the outpatients into four groups: those who had spine surgery (493 patients), those who had hip surgery (355 patients), those who had knee surgery (394 patients), and those who had surgery at other sites $(1,145$ patients).

SPSS ver. 18.0 (SPSS Inc., Chicago, IL, USA) was used for statistical analysis. The chi-square test was used to evaluate differences according to etiology. The results were deemed statistically significant if $p<0.05$.

\section{RESULTS}

Of the 3,611 patients, 172 patients (4.76\%) were diagnosed with delirium after orthopedic surgery. Postoperative delirium occurred in 15 patients $(1.18 \%)$ who were aged 50 to 59 years, 45 patients (3.86\%) aged 60 to 69 years, 76 patients $(8.49 \%)$ aged 70 to 79 years, and 36 patients $(13.04 \%)$ aged 80 years or older $(p<0.001)$ (Table 1). Of 2,538 patients who had orthopedic surgery under general anesthesia, 165 patients (6.50\%) were diagnosed with postoperative delirium. In contrast, five patients $(0.77 \%)$ who underwent spinal anesthesia and two patients $(0.47 \%)$ who underwent local anesthesia experienced postoperative delirium, a significant difference between groups $(p<0.001)$ (Table 2). Of 1,310 patients whose orthopedic surgery lasted more than 2 hours, 77 (5.88\%) were diagnosed with postoperative delirium; of 2,301 patients whose surgery lasted for less than 2 hours, 95 (4.13\%) were diagnosed with postoperative delirium, a significant difference between groups $(p=0.017$ ) (Table 3). Postoperative delirium was diagnosed in 100 patients $(8.17 \%)$ who were hospitalized through the emergency room and $72(3.02 \%)$ who were treated in an outpatient clinic $(p<0.001)$ (Table 4$)$.

$\begin{aligned} & \text { Table 3. Incidence of Postoperative Delirium According to Operation } \\
& \text { Time }\end{aligned}$
\begin{tabular}{ccc}
\hline Operation time & No. of patients & Postoperative delirium \\
\hline More than 2 hours & 1,310 & $77(5.88)$ \\
\hline Less than 2 hours & 2,301 & $95(4.13)$ \\
\hline
\end{tabular}

Values are presented as number (\%). 


$\begin{aligned} & \text { Table 4. Incidence of Postoperative Delirium According to Course } \\
& \text { of Hospitalization }\end{aligned}$
\begin{tabular}{cccc} 
Course of hospitalization & No. of patients & Postoperative delirium \\
\hline Emergency room & 1,224 & $100(8.17)$ \\
\hline Outpatient clinic & 2,387 & $72(3.02)$ \\
\hline
\end{tabular}

Values are presented as number (\%).

To determine the incidence of postoperative delirium in relation to the surgical site, we divided the patients into four groups who had surgery of the spine, hip, knee, or other site. Postoperative delirium occurred in 22 of 493 patients $(4.46 \%)$ after spine surgery, 18 of 355 patients (5.07\%) after hip surgery, 17 of 394 patients (4.31\%) after knee surgery, and 15 of 1,145 patients (1.31\%) after surgery at other sites $(p<0.001)$ (Table 5$)$.

\section{DISCUSSION}

As defined in the fourth edition of the Diagnostic and Statistical Manual of Mental Disorders, ${ }^{9,10)}$ delirium is "a disturbance of consciousness that is accompanied by a change in cognition that cannot be better accounted for by a preexisting or evolving dementia." The key characteristic is a change in mental status characterized by a reduced awareness of the environment and a disturbance in attention. ${ }^{9,10)}$ Delirium is a serious complication for elderly patients because it can result in functional decline, longer hospitalization, and institutionalization. ${ }^{6,11)}$ Patients who develop delirium during hospitalization have a higher risk of 6-month mortality than patients who do not develop delirium. Increased morbidity and mortality, longer hospital stay, cognitive and functional decline, and increased institutionalization can all increase social and health care cost. $^{6,11)}$ The risk of severe complications, such as a fall, myocardial infarction, pulmonary edema, pneumonia, and pressure ulcers, also increases in older patients with delirium. ${ }^{12)}$ Delirium may worsen the course and progress of dementia, leading to poorer long-term outcomes in terms of hospitalization time, care placement, and physical and cognitive functioning. ${ }^{13)}$

Postoperative delirium is a common geriatric complication, and its incidence ranges from $9 \%$ to $87 \%$ depending on both the patient population and the degree of operative stress. ${ }^{2,12)}$ The development of postoperative delirium is associated with multiple risk factors, such as preexisting dementia, old age, functional impairment, greater comorbidities, psychopathological symptoms, and

\begin{tabular}{lcc}
$\begin{array}{l}\text { Table 5. Incidence of Postoperative } \\
\text { According to Surgical Site }\end{array}$ & \\
\hline Surgical site & No. of patients & Postoperative delirium \\
\hline Spine & 493 & $22(4.46)$ \\
Hip & 355 & $18(5.07)$ \\
Knee & 394 & $17(4.31)$ \\
Others & 1,145 & $15(1.31)$ \\
\hline
\end{tabular}

Values are presented as number $(\%)$.

the degree of operative stress. ${ }^{2)}$ Of these preoperative risk factors, preexisting dementia appears to be the strongest predictor of postoperative delirium. ${ }^{2)}$

Nazemi et al. ${ }^{14)}$ reported that risk factors for delirium after elective spinal surgery include age, functional impairment, preexisting dementia, general anesthesia, surgical duration $>3$ hours, intraoperative hypercapnia and hypotension, greater blood loss, low hematocrit and albumin, preoperative affective dysfunction, and postoperative sleep disorders in their systemic review. We sought to determine whether patient age, type of anesthesia (general, spinal, or local), operation time, surgical site, and etiology (trauma or disease) were risk factors of postoperative delirium. We found that older patients were more vulnerable to postoperative delirium. Postoperative delirium was about 3.9 times more common in patients in their 70s and 80 s than patients in their 50 s and 60 s. Patients who underwent general anesthesia were about 10 times more likely to have postoperative delirium than patients who had local or spinal anesthesia. Patients whose surgery lasted more than 2 hours were about 1.4 times more likely to have postoperative delirium than patients whose surgical time was less than 2 hours.

We also divided the patients into two groups according to the course of hospitalization to compare patients who underwent surgery due to disease or trauma. We assumed that patients who underwent surgery due to disease were hospitalized through an outpatient clinic and that patients who underwent surgery due to trauma were hospitalized through the emergency room. Postoperative delirium was about 2.7 times more common in the patients hospitalized through the emergency room than in those hospitalized through an outpatient clinic.

The outpatients were further subdivided into four groups according to the surgical site (hip, knee, spine, and other site) to compare the incidence of postoperative delirium after surgery on different parts of the body. Since the trauma patients had multiple injuries to different parts 
of the body, it was not possible to assign them to just one group, so they were excluded from the analysis. Patients who had hip, knee, or spine surgery were about 3.5 times more likely to have postoperative delirium than patients who had surgery at other sites; postoperative delirium occurred most often in patients who had hip surgery.

Older patients are probably more vulnerable to postoperative delirium because their overall condition is generally poorer than that of younger patients, and they are more likely to have underlying disease. Given that postoperative delirium occurred more often in patients who underwent surgery under general anesthesia and those who had a surgery time over 2 hours, the use of anesthetic medicine may be associated with postoperative delirium. Because of the longer period without ambulation and the poorer general condition due to trauma, postoperative delirium was more likely to occur in patients hospitalized through the emergency room than in those hospitalized through an outpatient clinic.

Postoperative delirium was more common in the patients who underwent spine, hip, or knee surgery than in the patients who underwent surgery at other sites probably because the patients in the former group had greater blood loss and a longer period without ambulation. The older age and the longest period without ambulation of hip surgery patients may explain why postoperative delirium was most common in this group.

It is critical to take preventive measures for patients with a high risk of postoperative delirium and to take account of the serious complications associated with this condition, such as increased falls, myocardial infarction, pulmonary edema, pneumonia, and pressure ulcers. The method of prevention can be nonpharmacological or pharmacological. ${ }^{15,16)}$ Kostas et al. ${ }^{15)}$ suggested that nonpharmacological preventive measures should focus on 10 components: the oxygen supply of the central nervous system, fluid and electrolyte balance, pain management, reduction of unnecessary medications, adequate nutrition, regular bowel and bladder function, early mobilization and rehabilitation, prevention and management of postoperative complications, environmental stimuli, and appropriate delirium management. Postoperatively, decreasing the use of methylprednisolone and promoting movement with an appropriate orthosis can reduce the incidence of delirium. Polypharmacy is an independent risk factor for delirium and decreasing the use of delirium-inducing medications may reduce the incidence. ${ }^{14)}$

Other studies have noted the benefits of nonpharmacological prevention of delirium compared with standard management of patients at risk of developing this condition. The intervention consisted of the following six elements: education, provision of a clock, avoidance of sensory deprivation, presence of familiar objects in the room, reorientation of the patient provided by family members, and extended visitation times ( 5 hours daily). ${ }^{17)}$

In recent years, various pharmacological therapies have been investigated with respect to their possible role in the prevention and treatment of delirium. ${ }^{18)}$ For example, antipsychotics have been used widely in the pharmacological management of established delirium. Haloperidol and chlorpromazine are typical antipsychotic agents, which act as dopamine receptor antagonists. Atypical antipsychotic agents include olanzapine, risperidone, quetiapine, and ziprasidone. Alpha-receptor antagonists, such as dexmedetomidine and clonidine, produce sedation and analgesia without causing respiratory depression. Rivastigmine is an acetylcholinesterase and butyrylcholinesterase inhibitor indicated for Alzheimer's disease, Parkinson disease dementia, and Lewy-body dementia. ${ }^{16,18)}$ Nazemi et al. ${ }^{14)}$ performed systemic review about the management of postoperative delirium and showed that haloperidol is used widely to treat postoperative delirium. Randomized controlled trials show that adding quetiapine results in delirium resolution an average of 3.5 days faster than haloperidol alone $(p=0.001)$ and decreases agitation and length of stay ( $p=0.02$ and $p=0.05)$. Delirium is one of the consequences of impaired sleep. There is a strong association between sleep deprivation and delirium in both elderly patients after surgery and intensive care unit patients. Thus, factors that promote sleep may also prevent delirium. ${ }^{16)}$

This study has several limitations. It was a retrospective cohort study, and the number of risk factors investigated was not large. Furthermore, we assumed that patients with postoperative delirium were diagnosed and treated by psychiatrists after consultation. Therefore, patients who had mild symptoms of delirium and did not receive treatment by psychiatrists might have been excluded. To resolve this problem, prospective studies that evaluate additional risk factors, such as pre- and postoperative laboratory results, history of delirium, and postoperative complications, are required.

We found that postoperative delirium was more common in older patients who had surgery under general anesthesia, patients whose surgery lasted more than 2 hours, and patients who were hospitalized through the emergency room. Clinicians should recognize the risk factors of postoperative delirium and take measures to prevent and treat this condition in high risk groups. 


\section{CONFLICT OF INTEREST}

No potential conflict of interest relevant to this article was reported.

\section{ACKNOWLEDGEMENTS}

This work was supported by grant from Inje University in 2011.

\section{REFERENCES}

1. Bruce AJ, Ritchie CW, Blizard R, Lai R, Raven P. The incidence of delirium associated with orthopedic surgery: a meta-analytic review. Int Psychogeriatr. 2007;19(2):197-214.

2. Robinson TN, Eiseman B. Postoperative delirium in the elderly: diagnosis and management. Clin Interv Aging. 2008;3(2):351-5.

3. Bilotta F, Doronzio A, Stazi E, et al. Early postoperative cognitive dysfunction and postoperative delirium after anaesthesia with various hypnotics: study protocol for a randomised controlled trial. The PINOCCHIO trial. Trials. 2011;12:170.

4. Girard TD, Pandharipande PP, Ely EW. Delirium in the intensive care unit. Crit Care. 2008;12 Suppl 3:S3.

5. Kalisvaart KJ, de Jonghe JF, Bogaards MJ, et al. Haloperidol prophylaxis for elderly hip-surgery patients at risk for delirium: a randomized placebo-controlled study. J Am Geriatr Soc. 2005;53(10):1658-66.

6. Brauer C, Morrison RS, Silberzweig SB, Siu AL. The cause of delirium in patients with hip fracture. Arch Intern Med. 2000;160(12):1856-60.

7. Aziz KT, Best MJ, Naseer Z, et al. The association of delirium with perioperative complications in primary elective total hip arthroplasty. Clin Orthop Surg. 2018;10(3):286-91.

8. Tabet N, Howard R. Pharmacological treatment for the prevention of delirium: review of current evidence. Int J Geriatr Psychiatry. 2009;24(10):1037-44.

9. Deiner S, Silverstein JH. Postoperative delirium and cognitive dysfunction. Br J Anaesth. 2009;103 Suppl 1:i41-6.
10. Wass $\mathrm{S}$, Webster PJ, Nair BR. Delirium in the elderly: a review. Oman Med J. 2008;23(3):150-7.

11. Freter SH, Dunbar MJ, MacLeod H, Morrison M, MacKnight C, Rockwood K. Predicting post-operative delirium in elective orthopaedic patients: the Delirium Elderly At-Risk (DEAR) instrument. Age Ageing. 2005;34(2):169-71.

12. Flinn DR, Diehl KM, Seyfried LS, Malani PN. Prevention, diagnosis, and management of postoperative delirium in older adults. J Am Coll Surg. 2009;209(2):261-8.

13. Kagansky N, Rimon E, Naor S, Dvornikov E, Cojocaru L, Levy S. Low incidence of delirium in very old patients after surgery for hip fractures. Am J Geriatr Psychiatry. 2004;12(3):306-14.

14. Nazemi AK, Gowd AK, Carmouche JJ, Kates SL, Albert TJ, Behrend CJ. Prevention and management of postoperative delirium in elderly patients following elective spinal surgery. Clin Spine Surg. 2017;30(3):112-9.

15. Kostas TR, Zimmerman KM, Rudolph JL. Improving delirium care: prevention, monitoring, and assessment. Neurohospitalist. 2013;3(4):194-202.

16. Banh HL. Management of delirium in adult critically ill patients: an overview. J Pharm Pharm Sci. 2012;15(4):499-509.

17. Martinez FT, Tobar C, Beddings CI, Vallejo G, Fuentes P. Preventing delirium in an acute hospital using a non-pharmacological intervention. Age Ageing. 2012;41(5):629-34.

18. Wang J, Li Z, Yu Y, Li B, Shao G, Wang Q. Risk factors contributing to postoperative delirium in geriatric patients postorthopedic surgery. Asia Pac Psychiatry. 2015;7(4):37582. 